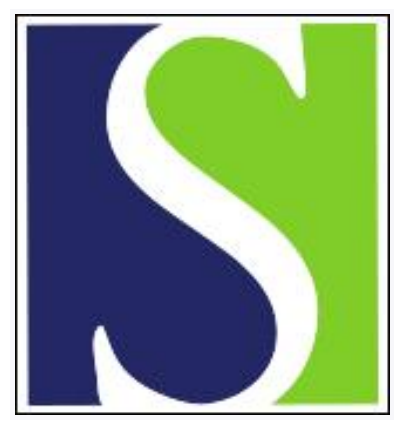

Scand J Work Environ Health 2013;39(6):559-567

https://doi.org/10.5271/sjweh.3370

Published online: 18 Jun 2013, Issue date: 01 Nov 2013

Association between shift work and periodontal health in a representative sample of an Asian population

by Han D-H, Khang Y-H, Jung-Choi K, Lim S

This study is the first step toward understanding the relationship between shift work and oral health. The results indicate that shift work might be associated with periodontitis. Therefore, it would be desirable to monitor shift workers' oral health status to identify high risk workers with different work schedules to increase their health.

Affiliation: Department of Preventive and Social Dentistry, School of Dentistry, Seoul National University, 101 Daehak-ro, Jongno-gu, Seoul 110-749, Korea.dhhan73@snu.ac.kr

Refers to the following texts of the Journal: 2012;38(4):343-348 2007;33(6):425-434 1999;25(6):610-615

The following article refers to this text: 2018;44(3):303-311

Key terms: Asia; epidemiology; inflammation; Korea; peridontal health; periodontitis; shift work

This article in PubMed: www.ncbi.nlm.nih.gov/pubmed/23780580

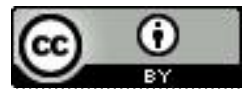




\title{
Association between shift work and periodontal health in a representative sample of an Asian population
}

\author{
by Dong-Hun Han, DDS, PhD, ${ }^{1,2}$ Young-Ho Khang, MD, PhD, ${ }^{3}$ Kyunghee Jung-Choi, MD, PhD, ${ }^{4}$ Sinye \\ Lim, MD, PhD ${ }^{5}$
}

\begin{abstract}
Han D-H, Khang Y-H, Jung-Choi K, Lim S. Association between shift work and periodontal health in a representative sample of an Asian population. Scand J Work Environ Health. 2013;39(6):559-567. doi:10.5271/sjweh.3370

Objective The association of shift work with periodontal disease is not well known. We studied the relationship between shift work and periodontitis in a representative sample of an Asian population.

Methods Participants were 4597 full-time employees from the Fourth Korean National Health and Nutrition Examination Survey (KNHANES IV). Shifting patterns were categorized into five shifts: daytime (N=3768), evening $(\mathrm{N}=121)$, night $(\mathrm{N}=59)$, rotating $(\mathrm{N}=206)$, and irregular $(\mathrm{N}=443)$. Periodontitis was measured with the Community Periodontal Index (CPI). Confounders included age, gender, income, and education. Mediators were frequency of daily tooth brushing, regular dental check-up, smoking, alcohol consumption, diabetes, obesity, and white blood cell count. Multiple logistic regression analyses were performed to evaluate the relationship.
\end{abstract}

Results The prevalence of periodontitis in the study population was $32.3 \%$. Shift workers had an elevated odds ratio (OR) of periodontitis [OR 1.22, 95\% confidence interval (95\% CI) 1.01-1.48] after controlling for confounders. The magnitude of the association between shift work and periodontitis attenuated with adjustment of mediators (smoking and a marker of inflammation). In subgroup analyses, the association was significant (OR $1.42,95 \%$ CI $1.03-1.95$ ) among those $\geq 45$ years. Irregular shift among those $\geq 45$ years showed a significant association (OR 1.79, 95\% CI 1.15-2.78).

Conclusion Findings of this cross-sectional study suggest that shift work may be associated with periodontitis. Shift workers aged $\geq 45$ years and irregular shift workers aged $\geq 45$ years were risk groups for periodontitis.

Key terms Asia; epidemiology; inflammation; Korea; periodontitis.

The management of work schedules (shift work) has become common in our $24 / 7$ society and increases night work and the diversity of flexible work-hour patterns in industrialized nations $(1,2)$. Approximately $15 \%$ of the fulltime workforce in the United States are shift workers (3). In the European Union (EU), $17 \%$ of the workforce are shift workers, while in the EU 27 countries, 19\% are night workers (4). In Asia, $22.7 \%$ and $15.5 \%$ are shiftworkers in Japan (5) and Taiwan (6), respectively. In Korea, shift workers represent $10.9 \%$ of the total workforce, and the shift patterns are regular 2 shifts (38.6\%), regular 3 shifts (23.9\%), 24-hour shift (14.0\%), irregular 2 shifts (5.7\%), fixed shifts (5.5\%), split shifts (3.6\%), irregular 3 shifts (2.9\%), and "other" (5.8\%) (7).

It is well known that several physiological functions follow a circadian rhythm, and long-term disturbance of the circadian rhythm reportedly has clinical consequences. Higher incidences of somatic diseases such as coronary heart disease (8) and metabolic syndrome (9), including obesity and diabetes (10), have been reported among shift workers. Circadian variations in immune defense and inflammation may also be affected by shift effects of occupational exposure. An increase in white blood cell (WBC) count among rotating shift compared

1 Department of Preventive and Social Dentistry, School of Dentistry, Seoul National University, Seoul, Korea.

2 Dental Research Institute, School of Dentistry, Seoul National University, Seoul, Korea.

3 Department of Preventive Medicine, University of Ulsan College of Medicine, Seoul, Korea.

4 Department of Preventive Medicine, Ewha Womans University School of Medicine, Seoul, Korea.

5 Department of Occupational and Environmental Medicine, Kyung Hee University Hospital, Seoul, Korea.

Correspondence to: Professor Dong-Hun Han, Department of Preventive and Social Dentistry, School of Dentistry, Seoul National University, 101 Daehak-ro, Jongno-gu, Seoul 110-749, Korea. [E-mail: dhhan73@snu.ac.kr] 
to daytime workers has been reported $(11,12)$. Elevated $\mathrm{C}$-reactive protein was associated with 3-shift work, and 2- and 3-shift work were related with increased WBC count (13).

Periodontal diseases are a group of bacterial inflammatory diseases of the supporting tissues of the teeth. Periodontal disease is one of the most common chronic inflammatory diseases in the world and occurs in at least $35 \%$ of the American population (14), 42.5\% among Japanese (15), and 33\% among Koreans (16). Moreover, periodontitis could be an early sign of severe inflammatory systemic disorder (17).

This study hypothesizes that shift work could be associated with periodontitis, and this association might be mediated by several potential mechanisms including health behaviors (oral health behaviors and other general health behaviors), somatic health problems (obesity and diabetes), and inflammation (measured by WBC count). To the best of our knowledge, there has been no study investigating the association between shift work and oral health. The aim of this study was to examine the relationship between shift work and periodontitis in a large representative sample of an Asian population: the Fourth Korea National Health and Nutritional Examination Survey (KNHANES IV).

\section{Methods}

\section{Study population}

Data were collected from the 2007-2009 stage of KNHANES IV, a cross-sectional and nationwide survey performed by the Korea Centers for Disease Control and Prevention (KCDC). KNHANES has been performed periodically since 1998 to investigate the health and nutritional status of Koreans. This survey employs a stratified, multi-stage, clustered probability sampling method to select a representative sample of the noninstitutionalized, civilian Korean population. The survey is composed of a health interview, a nutrition survey, and a health examination. Oral health has been included as part of the survey since the first year of KNHANES IV. Data were collected by household interviews and direct standardized physical examinations and fasting blood sampling conducted in mobile health examination centers. Each participant in the survey signed an informed consent form. Overall response rates were $65.8 \%$ in $2007,74.3 \%$ in 2008 , and $79.2 \%$ in 2009 .

The number of total participants in KNHANES IV was 24871 (11 310 males and 13561 females). Among those, periodontal health status was measured for 19091 participants (8404 males and 10687 females). Paid workers were 5787 (3089 males and 2698 females) among total participants in KNHANES IV ( $\mathrm{N}=24$ 871). Exclusion criteria were four-fold: (i) those aged $<25$ years, (ii) those aged $\geq 65$ years, (iii) the edentate (those with few teeth), and (iv) those missing values in the health assessment or questionnaires. The final sample size for our analysis was 4597 (2528 males and 2069 females (79.4\% of all paid workers in KNHANES IV).

\section{Assessment of shift work}

Work schedule was derived from the interview question, "Which of the following best describes the hours you usually work at this job?" There were eight possible responses: regular daytime schedule or shift; regular evening shift; regular night shift; rotating 12-hour shift; rotating 24-hour shift; split shift; irregular schedule; or "other". Shift work was defined as anything but a regular daytime schedule. Of these responses, four broad categories were used to define the type of shift work: evening, night, rotating, and irregular shifts. A rotating shift was defined as a rotating 8-, 12- or 24-hour shift. An irregular shift included split shift, irregular schedule, and "other" (18).

If a respondent asked for clarification of the various shift works at the interview, the following definitions were provided: A regular daytime schedule or shift refers to work beginning after $06: 00$ and ending before 18:00 hours. A regular evening shift refers to work beginning after 14:00 hours and ending before midnight. A regular night shift refers to work beginning after 21:00 and ending before 08:00 hours. A rotating shift changes periodically from days to evenings or nights. A split shift has two or more distinct periods each day.

\section{Assessment of periodontitis}

Dentists conducted an oral health examination in the survey. The World Health Organization (WHO) community periodontal index (CPI) was used to assess periodontitis (19). Periodontitis was defined as a CPI greater than or equal to "code 3", which indicates that more than one site had a $3.5 \mathrm{~mm}$ pocket or larger in the index teeth. The index teeth numbers were $11,16,17,26,27,31,36,37$, 46 , and 47 , respectively.

\section{Assessment of covariates}

Confounders in this study were major sociodemographic factors and included age, gender, income, and education. Mediators considered in the relationship between shift work and periodontitis were oral health behaviors (frequency of daily tooth brushing and regular dental check-up), general health behaviors (smoking and alcohol consumption), somatic health problems (obesity and diabetes), and a measure for inflammation (WBC count). 
We assumed these mediators as intermediate variables in the causal pathway between shift work and periodontitis. With the exception of obesity, diabetes, and WBC count, information on covariates was collected during the health interview. Education level was categorized into four groups: below primary school, middle school, high school, and college or higher. Monthly household income was adjusted for the number of household members and categorized into four groups: $<25,25-49$, $50-75$, and $>75 \%$ of total equalized income in the survey. Information on the frequency of daily tooth brushing was collected from the following question, "How often did you brush your teeth yesterday?" (times/day). Participants were asked whether they had visited a dental clinic for a regular check-up during a year prior to the interview (yes or no). Smoking status was divided into four categories: never smokers, ex-smokers, current smokers who smoked $<1$ pack per day, and current smokers who smoked $\geq 1$ pack per day. Alcohol consumption was assessed by questioning the participants about their average drinking frequency (days per month or week) during a year prior to the interview. Alcohol consumption status was categorized into five groups: non-drinker, almost non-drinker ( $\leq 1$ day per month), light drinker (2-4 days per month), moderate drinker ( $2-3$ days per week), and heavy drinker ( $\geq 4$ days per week). Obesity was measured based on standardized physical examination. Height and weight measurements were performed with the participants wearing light clothing and no shoes. Body mass index (BMI) was grouped as underweight $\left(<18.5 \mathrm{~kg} / \mathrm{m}^{2}\right)$, normal $(<25$ $\left.\mathrm{kg} / \mathrm{m}^{2}\right)$ and obese $\left(\geq 25 \mathrm{~kg} / \mathrm{m}^{2}\right)$. Fasting plasma glucose was measured and grouped as normal $(<100 \mathrm{mg} / \mathrm{dl})$, pre-diabetic $(<126 \mathrm{mg} / \mathrm{dl})$, and diabetic $(\geq 126 \mathrm{mg} / \mathrm{dl}$ or diabetic medication). WBC count was measured with blood specimens.

\section{Statistical analysis}

Individual sample weights and the complex sample design including stratification and primary sampling units were considered in the analysis.

As seen in figure 1, the role of confounders in the relationship between shift work (the independent variable of this study) and periodontitis (the dependent variable of this study) can be determined by examining the association of shift work with confounders (A in figure 1) and the association of confounders with periodontitis (B). Adjusted odds ratio $\left(\mathrm{OR}_{\mathrm{adj}}\right)$ of periodontitis adjusting for sociodemographic variables (age, gender, income, and education) represents the unconfounded total impact of shift work on periodontitis in this study. The relationship of mediators with shift work (C) and periodontitis (D) should also be examined to determine the role of mediators. This role of mediators was

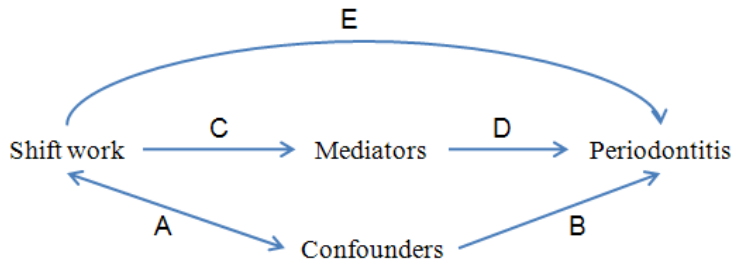

Figure 1. A model for the relationship between shift work and periodontitis and the roles of confounders and mediators in the relationship.

evaluated with the percentage (\%) excess odd explained, which can be calculated as $\left[\left(\mathrm{OR}_{\text {adj sociodemographic factors }}-\mathrm{OR}_{\mathrm{adj}}\right.\right.$ sociodemographic factors + mediators $\left.) /\left(\mathrm{OR}_{\text {adj sociodemographic factors }}-1\right)\right]$ in this study (20). This \% excess odd explained represents the degree to which a mediator explains the relationship between shift work and periodontitis.

In this study, the characteristics of study subjects by periodontitis status were presented with frequency distributions for the categorical variables and means (and standard deviations) for continuous variables (table 1). Chi-square tests for categorical variables and t-tests for continuous variables were used to assess the associations of periodontitis with shift work, confounders, and mediators. Age- and gender-adjusted OR of periodontitis were also presented to show the impact of these covariates on periodontitis. We then presented characteristics of study subjects according to shift work (table 2) and examined the association with Chi-square and t-tests. Series of multiple logistic regression analysis were used to estimate the $\mathrm{OR}_{\text {adj }}$ of periodontitis according to shift work after adjusting for mediator variables (table 3 ). The model adjusting for confounders was the base model (model 1 in table 3 ) in this analysis. Based on these analyses, $\%$ excess odd explained were calculated. We also presented findings with full adjustment for shift work and shift patterns (table 4). Due to the ageing difference between shift work and health outcome (21-23), the age-specific subgroup analyses were also performed (table 4).

\section{Results}

Of total study subjects $(\mathrm{N}=4597), 32.3 \%$ [95\% confidence interval (95\% CI) 30.4-34.2\%] had periodontitis $(\mathrm{CPI} \geq 3)$. Table 1 shows that shift workers $(\mathrm{N}=829)$ accounted for $18.6 \%$ (95\% CI $17.1-20.1 \%$ ) of total study population. According to the shift work patterns, evening shift workers accounted for $2.6 \%$ (95\% CI 2.1 $3.3 \%)$, night shift workers $1.3 \%$ (95\% CI $1.0-1.8 \%)$, rotating shift workers $4.5 \%$ (95\% CI 3.7-5.4\%), and 
Table 1. Characteristics of study subjects according to periodontal status: findings from Korea National Health and Nutrition Examination Survey, 2007-2009 ( $\mathrm{N}=4597$ ). Bold denotes statistical significance at $\mathbf{P}<\mathbf{0 . 0 5}$. [95\% $\mathrm{Cl}=\mathbf{9 5 \%}$ confidence interval]

\begin{tabular}{|c|c|c|c|c|c|c|c|c|c|c|c|}
\hline & \multicolumn{4}{|c|}{ No periodontitis ( $\mathrm{N}=3102)$} & \multicolumn{4}{|c|}{ Periodontitis $(\mathrm{N}=1495)$} & \multirow[b]{2}{*}{$P$-value a } & \multirow[b]{2}{*}{$\mathrm{OR}_{\mathrm{adj}}^{\mathrm{b}}$} & \multirow[b]{2}{*}{$95 \% \mathrm{Cl}$} \\
\hline & $\mathrm{N}$ & $\%$ & Mean & $95 \% \mathrm{Cl}$ & $\mathrm{N}$ & $\%$ & Mean & $95 \% \mathrm{Cl}$ & & & \\
\hline Age (years) & & & 38.02 & $37.57-38.47$ & & & 45.47 & $44.89-46.06$ & $<0.001^{c}$ & & \\
\hline \multicolumn{12}{|l|}{ Gender } \\
\hline Male $(\mathrm{N}=2528)$ & 1565 & 63.3 & & $60.9-65.7$ & 963 & 36.7 & & $34.3-39.1$ & $<0.001$ & & \\
\hline Female $(\mathrm{N}=2069)$ & 1537 & 75.1 & & $72.7-77.4$ & 532 & 24.9 & & $22.6-27.3$ & & & \\
\hline \multicolumn{12}{|l|}{ Shift work } \\
\hline No $(\mathrm{N}=3768)$ & 2567 & 68.6 & & $66.5-70.5$ & 1201 & 31.4 & & $29.5-33.5$ & 0.021 & 1.00 & reference \\
\hline Yes $(N=829)$ & 535 & 64.1 & & $60.3-67.7$ & 294 & 35.9 & & $32.3-39.7$ & & 1.27 & $1.05-1.53$ \\
\hline \multicolumn{12}{|l|}{ Shift pattern } \\
\hline Daytime $(\mathrm{N}=3768)$ & 2567 & 68.6 & & $66.5-70.5$ & 1201 & 31.4 & & $29.5-33.5$ & 0.035 & 1.00 & reference \\
\hline Evening $(\mathrm{N}=121)$ & 89 & 73.0 & & $63.2-81.0$ & 32 & 27.0 & & $19.0-36.8$ & & 1.22 & $0.76-1.96$ \\
\hline Night $(N=59)$ & 37 & 56.9 & & $41.6-71.0$ & 22 & 43.1 & & $29.0-58.4$ & & 2.00 & $0.92-4.33$ \\
\hline Rotating (N=206) & 120 & 60.6 & & $53.3-67.5$ & 86 & 39.4 & & $32.5-46.7$ & & 1.23 & $0.88-1.73$ \\
\hline Irregular $(\mathrm{N}=443)$ & 289 & 64.2 & & $59.1-69.1$ & 154 & 35.8 & & $30.9-40.9$ & & 1.22 & $0.96-1.56$ \\
\hline \multicolumn{12}{|l|}{ Income } \\
\hline I (N=1044) & 667 & 64.2 & & $60.8-67.5$ & 377 & 35.8 & & $32.5-39.2$ & $<0.001$ & 1.00 & reference \\
\hline$\|(N=1146)$ & 716 & 62.2 & & $58.7-65.6$ & 430 & 37.8 & & $34.4-41.3$ & & 1.03 & $0.82-1.28$ \\
\hline III $(\mathrm{N}=1186)$ & 832 & 70.8 & & $67.6-73.8$ & 354 & 29.2 & & $26.2-32.4$ & & 0.70 & $0.56-0.86$ \\
\hline IV $(\mathrm{N}=1221)$ & 887 & 72.7 & & $69.4-75.8$ & 334 & 27.3 & & $24.2-30.6$ & & 0.62 & $0.49-0.79$ \\
\hline \multicolumn{12}{|l|}{ Education } \\
\hline$\leq$ Primary school $(\mathrm{N}=531)$ & 256 & 45.7 & & $40.8-50.7$ & 275 & 54.3 & & $49.3-59.2$ & $<0.001$ & 1.00 & reference \\
\hline Middle school $(\mathrm{N}=436)$ & 239 & 54.6 & & $48.9-60.2$ & 197 & 45.4 & & $39.8-51.1$ & & 0.90 & $0.65-1.27$ \\
\hline High school (N=1719) & 1153 & 66.7 & & $63.8-69.4$ & 566 & 33.3 & & $30.6-36.2$ & & 0.96 & $0.72-1.28$ \\
\hline$\geq$ College $(\mathrm{N}=1911)$ & 1454 & 76.2 & & $73.9-78.5$ & 457 & 23.8 & & $21.5-26.1$ & & 0.65 & $0.48-0.88$ \\
\hline Tooth brushing (times/day) & & & 2.72 & $2.67-2.76$ & & & 2.51 & $2.46-2.57$ & $<0.001^{c}$ & 0.92 & $0.85-0.99$ \\
\hline \multicolumn{12}{|l|}{ Regular oral check-up } \\
\hline Yes $(N=1612)$ & 1134 & 70.0 & & $67.0-72.8$ & 478 & 30.0 & & $27.2-33.0$ & 0.025 & 1.00 & reference \\
\hline No $(\mathrm{N}=2985)$ & 1968 & 66.4 & & $64.3-68.5$ & 1017 & 33.6 & & $31.5-35.7$ & & 1.20 & $1.02-1.41$ \\
\hline \multicolumn{12}{|l|}{ Smoking } \\
\hline Never smoker $(\mathrm{N}=2444)$ & 1825 & 75.9 & & $73.6-78.0$ & 619 & 24.1 & & $22.0-26.4$ & $<0.001$ & 1.00 & reference \\
\hline Ex-smoker $(\mathrm{N}=781)$ & 462 & 59.8 & & $55.8-63.6$ & 319 & 40.2 & & $36.4-44.2$ & & 1.80 & $1.38-2.34$ \\
\hline Current smoker <1 pack/day $(\mathrm{N}=746)$ & 502 & 69.0 & & $65.3-72.5$ & 244 & 31.0 & & $27.5-34.7$ & & 1.87 & $1.48-2.36$ \\
\hline Current smoker $\geq 1$ pack/day $(\mathrm{N}=626)$ & 313 & 50.9 & & $46.5-55.4$ & 313 & 49.1 & & $44.6-53.5$ & & 3.29 & $2.50-4.33$ \\
\hline \multicolumn{12}{|l|}{ Current alcohol consumption } \\
\hline No $(\mathrm{N}=761)$ & 491 & 65.6 & & $61.5-69.5$ & 270 & 34.4 & & $30.5-38.5$ & $<0.001$ & 1.00 & reference \\
\hline$\leq 1$ time/month $(\mathrm{N}=1328)$ & 968 & 72.9 & & $69.9-75.8$ & 360 & 27.1 & & $24.2-30.1$ & & 0.96 & $0.76-1.21$ \\
\hline 2-4 times/month $(\mathrm{N}=1313)$ & 923 & 71.0 & & $67.8-74.0$ & 390 & 29.0 & & $26.0-32.2$ & & 1.04 & $0.83-1.32$ \\
\hline 2-3 times/week $(\mathrm{N}=909)$ & 584 & 64.3 & & $60.8-67.7$ & 325 & 35.7 & & $32.3-39.2$ & & 1.15 & $0.89-1.48$ \\
\hline$\geq 4$ times/week $(\mathrm{N}=286)$ & 136 & 48.0 & & $41.5-54.6$ & 150 & 52.0 & & $45.4-58.5$ & & 1.75 & $1.22-2.50$ \\
\hline Glucose (mg/dl) & & & 93.84 & $93.13-94.54$ & & & 99.25 & $97.93-100.58$ & $<0.001^{c}$ & 1.00 & $1.00-1.01$ \\
\hline \multicolumn{12}{|l|}{ Diabetes } \\
\hline Normal $(\mathrm{N}=3502)$ & 2477 & 70.9 & & $68.8-72.8$ & 1025 & 29.1 & & & $<0.001$ & 1.00 & reference \\
\hline Pre-diabetes $(\mathrm{N}=827)$ & 498 & 60.9 & & $56.9-64.8$ & 329 & 39.1 & & $35.2-43.1$ & & 1.03 & $0.85-1.25$ \\
\hline Diabetes $(\mathrm{N}=268)$ & 127 & 47.4 & & $40.8-54.0$ & 141 & 52.6 & & $46.0-59.2$ & & 1.21 & $0.89-1.64$ \\
\hline Body mass index $\left(\mathrm{kg} / \mathrm{m}^{2}\right)$ & & & 23.61 & $23.47-23.74$ & & 24.05 & & $23.86-24.24$ & $<0.001^{c}$ & 1.003 & $0.979-1.028$ \\
\hline \multicolumn{12}{|l|}{ Obesity } \\
\hline Underweight ( $\mathrm{N=184)}$ & 140 & 75.4 & & $68.0-81.5$ & 44 & 24.6 & & $18.5-32.0$ & $<0.001$ & 0.81 & $0.55-1.19$ \\
\hline Normal $(\mathrm{N}=2950)$ & 2028 & 68.8 & & $66.5-71.0$ & 922 & 31.2 & & $29.0-33.5$ & & 1.00 & reference \\
\hline Obesity $(\mathrm{N}=1463)$ & 934 & 64.8 & & $61.7-67.7$ & 529 & 35.2 & & $32.3-38.3$ & & 1.03 & $0.88-1.21$ \\
\hline White blood cell count & & & 6.17 & $6.10-6.25$ & & & 6.58 & $6.47-6.70$ & $<0.001^{c}$ & 1.15 & $1.10-1.20$ \\
\hline
\end{tabular}

a Obtained from chi-square test.

${ }^{\mathrm{b}}$ Age- and gender-adjusted odds ratio $\left(\mathrm{OR}_{\mathrm{adj}}\right)$ of periodontitis.

c Obtained from independent t-test. 
Table 2. Association of shift work and confounders and mediators: Findings from Korea Nation Health and Nutrition Examination Survey, 2007-2009 ( $\mathrm{N}=4597$ ). Bold denotes statistical significance at $\mathrm{P}<0.05$. Bold denotes statistical significance at $\mathbf{P}<\mathbf{0 . 0 5}$. $[95 \% \mathrm{Cl}=95 \%$ confidence interval]

\begin{tabular}{|c|c|c|c|c|c|c|c|c|c|}
\hline & \multicolumn{4}{|c|}{ Daytime work (N=3768) } & \multicolumn{4}{|c|}{ Shift work $(\mathrm{N}=829)$} & \multirow[t]{2}{*}{ P-value a } \\
\hline & $\mathrm{N}$ & $\%$ & Mean & $95 \% \mathrm{Cl}$ & $\mathrm{N}$ & $\%$ & Mean & $95 \% \mathrm{Cl}$ & \\
\hline Age (years) & & & 40.49 & $40.08-40.91$ & & & 40.13 & $39.32-40.94$ & $0.411^{b}$ \\
\hline \multicolumn{10}{|l|}{ Gender } \\
\hline Male $(\mathrm{N}=2528)$ & 2051 & 62.0 & & $60.4-63.7$ & 477 & 65.7 & & $62.0-69.2$ & \multirow[t]{2}{*}{0.085} \\
\hline Female (N=2069) & 1717 & 38.0 & & $36.3-39.6$ & 352 & 34.3 & & $30.8-38.0$ & \\
\hline \multicolumn{10}{|l|}{ Income } \\
\hline I - lowest (N=1044) & 845 & 21.9 & & $20.1-23.9$ & 199 & 24.1 & & $20.9-27.6$ & \multirow[t]{4}{*}{$<0.001$} \\
\hline II (N=1146) & 892 & 23.3 & & $21.6-25.0$ & 254 & 31.0 & & $27.5-34.6$ & \\
\hline III (N=1186) & 995 & 27.1 & & $25.5-28.9$ & 191 & 22.6 & & $19.3-26.3$ & \\
\hline IV - highest ( $(N=1221)$ & 1036 & 27.7 & & $25.4-30.1$ & 185 & 22.3 & & $18.9-26.2$ & \\
\hline \multicolumn{10}{|l|}{ Education } \\
\hline$\leq$ Primary school $(\mathrm{N}=531)$ & 432 & 9.4 & & $8.3-10.6$ & 99 & 10.1 & & $8.1-12.6$ & \multirow[t]{4}{*}{$<0.001$} \\
\hline Middle school $(\mathrm{N}=436)$ & 345 & 8.7 & & $7.7-9.9$ & 91 & 9.6 & & $7.6-12.1$ & \\
\hline High school $(\mathrm{N}=1719)$ & 1341 & 36.4 & & $34.3-38.5$ & 378 & 47.4 & & $43.7-51.2$ & \\
\hline$\geq$ College $(N=1911)$ & 1650 & 45.5 & & $42.9-48.0$ & 261 & 32.8 & & $29.2-36.6$ & \\
\hline Tooth brushing (times/day) & & & 2.65 & $2.61-2.69$ & & & 2.66 & $2.57-2.75$ & $0.800^{b}$ \\
\hline \multicolumn{10}{|l|}{ Regular oral check-up } \\
\hline Yes $(N=1612)$ & 1332 & 36.7 & & $34.6-38.8$ & 280 & 35.2 & & $31.3-39.4$ & \multirow[t]{2}{*}{0.469} \\
\hline No $(\mathrm{N}=2985)$ & 2436 & 63.3 & & $61.2-65.4$ & 549 & 64.8 & & $60.6-68.7$ & \\
\hline \multicolumn{10}{|l|}{ Smoking } \\
\hline Never smoker $(\mathrm{N}=2444)$ & 2028 & 48.3 & & $46.6-49.9$ & 416 & 43.6 & & $39.8-47.4$ & \multirow[t]{4}{*}{0.016} \\
\hline Ex-smoker $(\mathrm{N}=781)$ & 633 & 18.4 & & $17.1-19.7$ & 148 & 18.6 & & $15.9-21.7$ & \\
\hline Current smoker <1pack/day $(\mathrm{N}=746)$ & 618 & 18.6 & & $17.2-20.1$ & 128 & 18.3 & & $15.5-21.4$ & \\
\hline Current smoker $\geq 1$ pack/day $(\mathrm{N}=626)$ & 489 & 14.7 & & $13.5-16.1$ & 137 & 19.6 & & $16.7-22.7$ & \\
\hline \multicolumn{10}{|l|}{ Current alcohol consumption } \\
\hline No $(N=761)$ & 621 & 14.7 & & $13.4-16.0$ & 140 & 15.7 & & $13.2-18.5$ & \multirow[t]{5}{*}{0.552} \\
\hline$\leq 1$ time/month $(\mathrm{N}=1328)$ & 1093 & 26.9 & & $25.3-28.5$ & 235 & 26.8 & & $23.2-30.6$ & \\
\hline $2-4$ times/month $(\mathrm{N}=1313)$ & 1092 & 30.5 & & $28.7-32.2$ & 221 & 27.5 & & $24.3-30.8$ & \\
\hline 2-3 times/week $(\mathrm{N}=909)$ & 728 & 21.3 & & $19.9-22.9$ & 181 & 23.2 & & $20.2-26.6$ & \\
\hline$\geq 4$ times/week $(\mathrm{N}=286)$ & 234 & 6.7 & & $5.8-7.6$ & 52 & 6.8 & & $5.2-8.9$ & \\
\hline Glucose (mg/dl) & & & 95.98 & $95.26-96.70$ & & & 93.86 & $92.61-95.11$ & $0.003^{b}$ \\
\hline \multicolumn{10}{|l|}{ Diabetes } \\
\hline Normal (N=3502) & 2844 & 75.5 & & $73.9-77.0$ & 658 & 80.1 & & $76.8-83.0$ & \multirow[t]{3}{*}{0.028} \\
\hline Pre-diabetes (N=827) & 702 & 18.7 & & $17.3-20.1$ & 125 & 14.3 & & $11.9-17.1$ & \\
\hline Diabetes $(\mathrm{N}=268)$ & 222 & 5.8 & & $5.0-6.8$ & 46 & 5.6 & & $4.1-7.7$ & \\
\hline Body mass index (kg/m2) & & & 23.74 & $23.62-23.86$ & & & 23.78 & $23.52-24.04$ & 0.794 b \\
\hline \multicolumn{10}{|l|}{ Obesity } \\
\hline Underweight ( $\mathrm{N=184)}$ & 145 & 3.7 & & $3.1-4.5$ & 39 & 4.3 & & $3.1-6.1$ & \multirow[t]{3}{*}{0.730} \\
\hline Normal $(N=2950)$ & 2423 & 63.2 & & $61.4-64.9$ & 527 & 63.2 & & $59.3-66.8$ & \\
\hline Obesity ( $N=1463)$ & 1200 & 33.1 & & $31.4-34.9$ & 263 & 32.5 & & $28.9-36.4$ & \\
\hline White blood cell count & & & 6.24 & $6.17-6.31$ & & & 6.59 & $6.43-6.75$ & $<0.001^{b}$ \\
\hline
\end{tabular}

a Obtained from chi-square test.

${ }^{\mathrm{b}}$ Obtained from independent t-test.

irregular shift workers $10.1 \%(95 \%$ CI $8.8-11.6 \%)$. Significant differences in the distribution of periodontitis were found with regards to all variables (all P-values $<0.05$ ). Except for diabetes, BMI, and obesity, significant age- and gender-adjusted OR of other variables were presented (table 1). Shift work had a significant age- and gender-adjusted $\mathrm{OR}\left(\mathrm{OR}_{\text {adj }} 1.27,95 \%\right.$ CI $1.05-$ $1.53)$. Night shift showed a relatively great $\mathrm{OR}_{\text {adj }}$ of 2.00 (95\% CI $0.92-4.33)$ of periodontitis.

Table 2 shows the relationship of shift work with confounders and mediators. Shift work was not pat- terned by age, gender and frequency of daily tooth brushing $(\mathrm{P}>0.05)$. However, shift workers were more likely to have lower income and lower education (all P-values $<0.001$ ). There were also significant differences in the distribution of shift work with regards to smoking, blood glucose level, diabetes, and WBC count (table 2).

Table 3 shows series of $\mathrm{OR}_{\mathrm{adj}}$ of periodontitis by shift work and shift work patterns after adjusting for mediators. Model 1 was adjusted for confounders (age, gender, income, and education). The analysis results revealed that shift workers had significantly higher $\mathrm{OR}_{\mathrm{adj}}$ 
Table 3. Roles of mediators in the relationship between shift work and periodontitis: Adjusted odds ratio $\left(0 \mathrm{R}_{\mathrm{adj}}\right)$ of periodontitis by shift work and working pattern and percentage excess odd explained ( $\mathrm{N}=4597)$. [WBC=white blood cell count]

\begin{tabular}{|c|c|c|c|c|c|c|c|c|c|c|c|c|c|c|c|}
\hline & \multicolumn{3}{|c|}{ Shift work (reference=no) } & \multicolumn{12}{|c|}{ Shift pattern (reference=daytime) } \\
\hline & \multicolumn{2}{|r|}{$\begin{array}{c}\text { Yes } \\
(\mathrm{N}=829)\end{array}$} & \multirow{2}{*}{$\begin{array}{c}\% \\
\text { excess } \\
\text { odd } \\
\text { explained } \\
\end{array}$} & \multicolumn{2}{|c|}{$\begin{array}{l}\text { Evening } \\
(\mathrm{N}=121)\end{array}$} & \multirow{2}{*}{$\begin{array}{c}\% \\
\text { excess } \\
\text { odd } \\
\text { explained }\end{array}$} & \multicolumn{2}{|r|}{$\begin{array}{c}\text { Night } \\
(\mathrm{N}=59)\end{array}$} & \multirow{2}{*}{$\begin{array}{c}\% \\
\text { excess } \\
\text { odd } \\
\text { explained }\end{array}$} & \multicolumn{2}{|c|}{$\begin{array}{l}\text { Rotating } \\
(\mathrm{N}=206)\end{array}$} & \multirow{2}{*}{$\begin{array}{c}\% \\
\text { excess } \\
\text { odd } \\
\text { explained }\end{array}$} & \multicolumn{2}{|c|}{$\begin{array}{l}\text { Irregular } \\
(\mathrm{N}=443)\end{array}$} & \multirow{2}{*}{$\begin{array}{c}\% \\
\text { excess } \\
\text { odd } \\
\text { explained }\end{array}$} \\
\hline & $O \mathrm{R}_{\mathrm{adj}}$ & dj $\quad 95 \% \mathrm{Cl}$ & & $O R_{a d j}$ & $95 \% \mathrm{Cl}$ & & $\mathrm{d}_{\mathrm{adj}}$ & $95 \% \mathrm{Cl}$ & & $d O R_{\text {adj }}$ & $95 \% \mathrm{Cl}$ & & $d O R_{a d j}$ & $95 \% \mathrm{Cl}$ & \\
\hline Model $1^{\mathrm{a}}$ & 1.22 & $1.01-1.48$ & & 1.17 & $0.73-1.88$ & & 1.75 & $0.80-3.83$ & & 1.21 & $0.86-1.70$ & & 1.18 & $0.93-1.51$ & \\
\hline $\begin{array}{l}\text { Model } 1 \\
+ \text { tooth } \\
\text { brushing }\end{array}$ & 1.23 & $1.01-1.48$ & -4.5 & 1.15 & $0.72-1.86$ & 11.8 & 1.74 & $0.79-3.85$ & 1.3 & 1.22 & $0.86-1.71$ & -4.8 & 1.19 & $0.93-1.52$ & -5.6 \\
\hline $\begin{array}{l}\text { Model } 1+ \\
\text { regular den- } \\
\text { tal check-up }\end{array}$ & 1.22 & $1.01-1.48$ & 0.0 & 1.15 & $0.72-1.86$ & 11.8 & 1.73 & $0.79-3.78$ & 2.7 & 1.21 & $0.86-1.71$ & 0.0 & 1.19 & $0.93-1.52$ & -5.6 \\
\hline $\begin{array}{l}\text { Model } 1+ \\
\text { smoking }\end{array}$ & 1.21 & $1.00-1.46$ & 4.5 & 1.14 & $0.70-1.85$ & 17.6 & 1.59 & $0.72-3.51$ & 21.3 & 1.21 & $0.85-1.71$ & 0.0 & 1.18 & $0.92-1.51$ & 0.0 \\
\hline $\begin{array}{l}\text { Model } 1 \\
+ \text { alcohol } \\
\text { consumption }\end{array}$ & 1.23 & $1.02-1.49$ & -4.5 & 1.16 & $0.72-1.85$ & 5.9 & 1.72 & $0.79-3.75$ & 4.0 & 1.22 & $0.87-1.72$ & -4.8 & 1.19 & $0.94-1.52$ & -5.6 \\
\hline $\begin{array}{l}\text { Model } 1+ \\
\text { diabetes }\end{array}$ & 1.23 & $1.01-1.49$ & -4.5 & 1.18 & $0.74-1.90$ & -5.9 & 1.76 & $0.80-3.85$ & -1.3 & 1.21 & $0.86-1.70$ & 0.0 & 1.19 & $0.93-1.52$ & -5.6 \\
\hline $\begin{array}{l}\text { Model } 1+ \\
\text { obesity }\end{array}$ & 1.22 & $1.01-1.48$ & 0.0 & 1.17 & $0.73-1.88$ & 0.0 & 1.75 & $0.80-3.81$ & 0.0 & 1.20 & $0.86-1.69$ & 4.8 & 1.18 & $0.93-1.51$ & 0.0 \\
\hline $\begin{array}{l}\text { Model } 1+ \\
\text { WBC count }\end{array}$ & 1.18 & $0.97-1.44$ & 18.2 & 1.14 & $0.71-1.82$ & 17.6 & 1.65 & $0.77-3.52$ & 13.3 & 1.16 & $0.82-1.64$ & 23.8 & 1.16 & $0.90-1.48$ & 11.1 \\
\hline
\end{tabular}

a Multivariate model adjusted for age (continuous), gender, income and education.

Table 4. Fully adjusted a ${ }^{2}$ dds ratios $\left(\mathrm{OR}_{\mathrm{adj}}\right)$ of periodontitis by shift work: Findings from Korea Nation Health and Nutrition Examination Survey, 2007-2009 ( $\mathrm{N}=4597)$. Bold denotes statistical significance at $\mathbf{P}<\mathbf{0 . 0 5}$. [95\% $\mathrm{Cl}=95 \%$ confidence interval]

\begin{tabular}{|c|c|c|c|c|c|c|c|c|c|c|c|c|c|c|}
\hline & \multicolumn{6}{|c|}{ Shift work (reference=no) } & \multicolumn{8}{|c|}{ Shift pattern (reference=daytime) } \\
\hline & \multicolumn{2}{|c|}{$\begin{array}{c}\text { No } \\
(\mathrm{N}=3768)\end{array}$} & \multicolumn{2}{|c|}{$\begin{array}{c}\text { Yes } \\
(\mathrm{N}=829)\end{array}$} & \multicolumn{2}{|c|}{$\begin{array}{c}\text { Daytime } \\
(\mathrm{N}=3768)\end{array}$} & \multicolumn{2}{|c|}{$\begin{array}{l}\text { Evening } \\
(\mathrm{N}=121)\end{array}$} & \multicolumn{2}{|c|}{$\begin{array}{l}\text { Night } \\
(\mathrm{N}=59)\end{array}$} & \multicolumn{2}{|c|}{$\begin{array}{l}\text { Rotating } \\
(\mathrm{N}=206)\end{array}$} & \multicolumn{2}{|c|}{$\begin{array}{l}\text { Irregular } \\
(\mathrm{N}=443)\end{array}$} \\
\hline & $\mathrm{OR}_{\mathrm{adj}}$ & $95 \% \mathrm{Cl}$ & $\mathrm{OR}_{\mathrm{adj}}$ & $95 \% \mathrm{Cl}$ & $\mathrm{OR}_{\mathrm{adj}}$ & $95 \% \mathrm{Cl}$ & $\mathrm{OR}_{\mathrm{adj}}$ & $95 \% \mathrm{Cl}$ & $\mathrm{OR}_{\mathrm{adj}}$ & $95 \% \mathrm{Cl}$ & $\mathrm{OR}_{\mathrm{adj}}$ & $95 \% \mathrm{Cl}$ & $\overline{O R_{a d j}}$ & $95 \% \mathrm{Cl}$ \\
\hline $\begin{array}{l}\text { Total } \\
(\mathrm{N}=4597)\end{array}$ & 1.00 & reference & 1.19 & $0.98-1.44$ & 1.00 & reference & 1.11 & $0.69-1.79$ & 1.54 & $0.72-3.27$ & 1.17 & $0.82-1.66$ & 1.17 & $0.92-1.51$ \\
\hline \multicolumn{15}{|c|}{ Age subgroup } \\
\hline$<45$ years & 1.00 & reference & 1.09 & $0.84-1.42$ & 1.00 & reference & 0.81 & $0.41-1.60$ & 2.32 & $0.98-5.49$ & 1.40 & $0.93-2.10$ & 0.93 & $0.66-1.31$ \\
\hline$\geq 45$ years & 1.00 & reference & 1.42 & $1.03-1.95$ & 1.00 & reference & 1.85 & $0.66-5.20$ & 0.65 & $0.21-2.01$ & 0.96 & $0.56-1.65$ & 1.79 & $1.15-2.78$ \\
\hline
\end{tabular}

a Model was adjusted for age (continuous), gender, income, education, frequency of daily tooth brushing (continuous), smoking, drinking, regular dental check-up, diabetes, obesity and white blood cell count.

than daytime workers in the age, gender, income and education-adjusted model (1.22, 95\% CI 1.01-1.48). The magnitude of $\mathrm{OR}_{\mathrm{adj}}$ of periodontitis by shift work attenuated with adjustment for smoking and a marker of inflammation (WBC count). The \% excess odd explained was higher for WBC count than others $(18.2 \%$ versus $-4.5 \%$ for tooth brushing, $0.0 \%$ for regular dental checkup, $4.5 \%$ for smoking, $-4.5 \%$ for alcohol consumption, $-4.5 \%$ for diabetes, and $0.0 \%$ for obesity).

Table 3 also presents the findings by shift pattern. The $\%$ excess odd explained was generally higher when WBC count was adjusted in the model. This was true for evening (17.6\%), rotating $(23.8 \%)$, and irregular $(11.1 \%)$ shift work. In the evening and night shifts, the role of smoking was also important in explaining the relationship between shift pattern and periodontitis. A total of $17.6 \%$ (for evening shift) and $21.3 \%$ (for night shift) excess odd in the relationship of shift pattern and periodontitis was explained by smoking. Although night shift showed the highest $\mathrm{OR}_{\text {adj }}$ among the shift work pattern, there was no significant $\mathrm{OR}_{\text {adj }}$ of periodontitis by shift work pattern.

Table 4 shows the $\mathrm{OR}_{\mathrm{adj}}$ of periodontitis in the fully adjusted model. The magnitude of the relationship between shift work and periodontitis attenuated after controlling for all covariates and was not statistically significant $\left(\mathrm{OR}_{\mathrm{adj}} 1.19,95 \% \mathrm{CI} 0.98-1.44\right)$.

In the age-specific subgroup analyses (table 4), night shift showed a very high likelihood of having periodontitis among the younger age group (those aged 
$<45$ years), although it was not significant. Shift workers aged $\geq 45$ years were more likely to have periodontitis than daytime workers in the same age group $\left(\mathrm{OR}_{\text {adj }} 1.42\right.$, 95\% CI 1.03-1.95). Moreover, in this older age group, irregular shift workers showed a significant higher odd of periodontitis than other types of shift workers $\left(\mathrm{OR}_{\mathrm{adj}}\right.$ $1.79,95 \%$ CI 1.15-2.78), while night shift did not show any tendency of increased odd of periodontitis.

\section{Discussion}

Results of this study identified an epidemiological association between shift work and periodontitis in a representative Korean sample. Analysis results showed that shift workers had a $27 \%$ greater odd of having periodontitis than daytime workers in an age- and genderadjusted model. This association modestly attenuated with further adjustment of socioeconomic status (income and education) but remain statistically significant $\left(\mathrm{OR}_{\mathrm{adj}}\right.$ $1.22,95 \%$ CI 1.01-1.48). It is well established that work and the workplace are important social determinants of health (24). Shift work is associated with coronary heart disease (8). Insomnia, a complaint common among night shift workers, is an independent risk factor for myocardial infarction (25). Periodontitis shares common risk factors with metabolic syndrome (26), diabetes mellitus (27), and hypertension (28). Inflammation plays a critical role in the atherosclerotic process (29) and pathogenesis of periodontitis (30). If periodontitis and other systemic diseases could be linked via inflammation, working condition could be considered of risk factors for periodontitis.

In our analysis, we tested the "inflammation" hypothesis with WBC count. Our results showed that WBC count played a role in explaining the relationship between shift work and periodontitis. The \% excess odd explained by WBC $(18.2 \%)$ was higher than the \% excess odd explained by other mediators. This was generally true for the relationship between shift pattern and periodontitis. Circadian variations in inflammation may be affected by shift work as WBC count (11-13) increases. Our study suggests that shift work may increase peripheral inflammation measured by WBC count, and WBC count is a relatively independent risk factor.

Smoking showed a dose-response association with periodontitis in our study (table 1), which corroborates a previous finding (31). Moreover, results of this study showed that shift workers were more heavy smokers (current smoker $\geq 1 \mathrm{pack} /$ day) than daytime workers $(\mathrm{P}=0.016$, table 2). Prior studies also presented that shift workers had higher smoking rates than daytime workers $(32,33)$. In our results, smoking is the second strongest impact on the relationship between shift work and peri- odontitis and the strongest impact on the relationship between night shift and periodontitis, which supported our hypothesis.

On the other hand, alcohol consumption was not associated with shift work in our study (table 2) and showed negligible $\%$ excess odd explained in the relationship between shift work and periodontitis (table $3)$. Many prior studies could not find differences in alcohol intake between daytime and shift workers (34, $35)$, although there are some exceptions $(32,36)$. Time constraints among shift workers might have prevented them from indulging in excessive alcohol consumption, while this would not be the case for smoking. However, detailed mechanisms should be further investigated as to why significant differences in alcohol consumption were not found between daytime and shift workers.

Oral health behavior, frequency of tooth brushing, and regular dental check-up played limited roles in the relationship between shift work and periodontitis in this study. Even though these oral health behaviors predicted the periodontitis (table 1), the differences in these behaviors according to shift work disappeared.

There is considerable epidemiological evidence that shift work is associated with increased risk for obesity and diabetes, perhaps as a result of physiological maladaptation to chronically sleeping and eating at abnormal circadian times. The impact of shift work on metabolism supports a possible pathway to the development of obesity and diabetes (37). However, we could not find an association between shift work and obesity/ diabetes and an association between periodontitis and obesity/diabetes.

Our results showed a significant association between shift work and periodontitis among those $\geq 45$ years $\left(\mathrm{OR}_{\mathrm{adj}} 1.44,95 \% \mathrm{CI} 1.05-1.97\right)$. Studies on ageing and shift work show conflicting results. The majority of studies on this topic conclude that tolerance for shift work decreases with age, and that increasing age is associated with increasing negative health effects of shift work (22, 23 ). The critical age for reduced tolerance to shift and night work is reported to be between $40-50$ years (22, 38). The positive association between shift work/irregular shift and periodontitis among the $\geq 45$-year old group might support the above studies. However, night shift work showed the strongest, but non-significant association with periodontitis among the $<45$-year old group in this study $\left(\mathrm{OR}_{\text {adj }} 2.34,95 \%\right.$ CI $\left.0.98-5.60\right)$. Some have argued that shift work could be better tolerated with older age, and younger people can find it more difficult to adapt to night work due to higher sensitivity to acute sleep loss than older people and because shift work hampers the possibility to participate and integrate in social life (21). Findings indicating that the negative health effects of shift work seem to increase with age might also be explained by factors other than age per se, such 
as total shift work exposure. Due to the conflicting view on how age is related to shift work tolerance, further studies are needed in different shift work populations and groups with different work schedules.

Although this is the first study to explore the relationship between shift work and periodontitis from the representative national Korean sample, it had some limitations. First, because the duration of shift work was not included in the model, the results of our study, such as the positive association between shift work and periodontitis among $\geq 45$-year-olds, could be biased. The lack of information about the shift work period might be larger among the older than younger workers because the past shift working history was not obtained. Second, irregular shift workers include split shift, irregular schedule, and "other". Among the respondents in irregular shift workers ( $\mathrm{N}=443)$, "other" $=352$. A misclassification bias in using the irregular shift work category could affect both the magnitude and direction of the observed associations of this study. Third, the cross-sectional design prohibited us from inferring causal relationships. Further well-designed prospective investigations are required to determine the causality between shift work and periodontitis to reduce the above-mentioned limitations. Fourth, in addition, the healthy worker effect may have influenced our results. The healthy worker effect states that those who stay in shift work are survivors who can withstand the strenuous shift work and that those who move into daytime work are likely to have a worse health status. Thus, the healthy worker effect may have resulted in an under- rather than overestimation of the effects of shift work.

There is a reason to believe that shift work may become a major occupational oral health and safety problem in the near future. The prevention of shiftworkrelated oral health and safety problems will be a major challenge for the employer, employees, and occupational oral health professionals during the next few decades. Our findings have several implications. The increased risk of periodontitis apparent among shift workers suggests that people who do shift work should be vigilant about risk factor modification. Screening programs for periodontal risk factors among shift workers would have yielded substantial help of treatable risks, including dyslipidaemia, hypertension, and cardiovascular diseases. Shift workers should be educated about oral symptoms in an effort to forestall or avert the earliest clinical manifestations of disease. Evidence also exists in the literature to suggest that modification and rationalization of shift schedules may yield dividends in terms of healthier, more productive workers; however, the long-term effects of these alterations on periodontal outcomes remain unknown. There is little evidence for a causal relationship between shift work and periodontitis. To demonstrate causality, we also need studies on mechanisms. We need more prospective studies with large representative populations, good descriptions of the working hours used, long follow-ups, and reliable measurements of health outcomes. More work is needed to identify the most vulnerable subsets of shift workers and the effects of shift modifying strategies on overall oral health.

\section{References}

1. Costa G. The 24-hour society between myth and reality. J Hum Ergol (Tokyo). 2001;30:15-20.

2. Harma MI, Ilmarinen JE. Towards the 24-hour society--new approaches for aging shift workers? Scand J Work Environ Health. 1999;25:610-5. http://dx.doi.org/10.5271/sjweh.488.

3. McMenamin TM. Time to work: Recent trends in shift work and flexible schedules. Monthly Lab Rev. 2007;130:3-15.

4. Eurofound. Fifth european working conditions survey: Overview report. Luxembourg: Publications Office of the European Union; 2012.

5. Ministry of Health, Labour and Welfare in Japan. General survey on working conditions in 2005. Tokyo: Ministry of Health, Labour and Welfare in Japan; 2005.

6. Cheng WJ, Cheng Y, Huang MC, Chen CJ. Alcohol dependence, consumption of alcoholic energy drinks and associated work characteristics in the Taiwan working population. Alcohol Alcoholism. 2012;47:372-79. http:// dx.doi.org/10.1093/alcalc/ags034.

7. Institute of Occupational Safety and Health Research. Second Korean working conditions survey. Incheon: Korea Occupational Safety and Health Agency; 2010.

8. Virkkunen H, Harma M, Kauppinen T, Tenkanen L. Shift work, occupational noise and physical workload with ensuing development of blood pressure and their joint effect on the risk of coronary heart disease. Scand J Work Environ Health. 2007;33:425-34. http://dx.doi.org/10.5271/sjweh.1170.

9. Puttonen S, Viitasalo K, Harma M. The relationship between current and former shift work and the metabolic syndrome. Scand J Work Environ Health. 2012;38:343-8. http://dx.doi. org/10.5271/sjweh.3267.

10. Pan A, Schernhammer ES, Sun Q, Hu FB. Rotating night shift work and risk of type 2 diabetes: Two prospective cohort studies in women. PLoS Med. 2011;8:e1001141. http://dx.doi. org/10.1371/journal.pmed.1001141.

11. Nishitani N, Sakakibara H. Subjective poor sleep and white blood cellcountinmalejapaneseworkers. IndHealth. 2007;45:296-300. http://dx.doi.org/10.2486/indhealth.45.296.

12. Sookoian S, Gemma C, Fernandez Gianotti T, Burgueno A, Alvarez A, Gonzalez CD, et al. Effects of rotating shift work on biomarkers of metabolic syndrome and inflammation. J Intern Med. 2007;261:285-92. http://dx.doi.org/10.1111/ j.1365-2796.2007.01766.x.

13. Puttonen S, Viitasalo K, Harma M. Effect of shiftwork 
on systemic markers of inflammation. Chronobiol Int 2011;28:528-35. http://dx.doi.org/10.3109/07420528.2011. 580869 .

14. Albandar JM, Brunelle JA, Kingman A. Destructive periodontal disease in adults 30 years of age and older in the united states, 1988-1994. J Periodontol. 1999;70:13-29. http:// dx.doi.org/10.1902/jop.1999.70.1.13.

15. Ojima M, Hanioka T, Tanaka K, Inoshita E, Aoyama H. Relationship between smoking status and periodontal conditions: Findings from national databases in japan. J Periodontal Res. 2006;41:573-9. http://dx.doi.org/10.1111/ j.1600-0765.2006.00915.x.

16. Kwon YE, Ha JE, Paik DI, Jin BH, Bae KH. The relationship between periodontitis and metabolic syndrome among a korean nationally representative sample of adults. J Clin Periodontol. 2011;38:781-6. http://dx.doi.org/10.1111/ j.1600-051X.2011.01756.x.

17. D'Aiuto F, Nibali L, Parkar M, Patel K, Suvan J, Donos N. Oxidative stress, systemic inflammation, and severe periodontitis. J Dent Res. 2010;89:1241-46. http://dx.doi. org/10.1177/0022034510375830.

18. Shields M. Shift work and health. Health Rep. 2002;13:11-33.

19. Ainamo J, Barmes D, Beagrie G, Cutress T, Martin J, Sardoinfirri J. Development of the World Health Organization (who) community periodontal index of treatment needs (CPITN). Int Dent J. 1982;32:281-91.

20. Szklo M, Nieto J. Epidemiology: Beyond the basics. 2nd ed. London: Jones \& Bartlett Publishers; 2008.

21. Costa G. Shift work and occupational medicine: An overview. Occup Med (Lond). 2003;53:83-8. http://dx.doi.org/10.1093/ occmed/kqg045.

22. Harma MI, Hakola T, Akerstedt T, Laitinen JT. Age and adjustment to night work. Occup Environ Med. 1994;51:56873. http://dx.doi.org/10.1136/oem.51.8.568.

23. Saksvik IB, Bjorvatn B, Hetland H, Sandal GM, Pallesen S. Individual differences in tolerance to shift work--a systematic review. Sleep Med Rev. 2011;15:221-35. http://dx.doi. org/10.1016/j.smrv.2010.07.002.

24. Theorell T. Working conditions and health. In: Social epidemiology. Berkman LF, Kawachi I, editors. New York: Oxford University Press; 2000.

25. Laugsand LE, Vatten LJ, Platou C, Janszky I. Insomnia and the risk of acute myocardial infarction: A population study. Circulation. 2011;124:2073-81. http://dx.doi.org/10.1161/ CIRCULATIONAHA.111.025858.

26. Han DH, Lim S, Paek D, Kim HD. Periodontitis could be related factors on metabolic syndrome among koreans: A casecontrol study. J Clin Periodontol .2012;39:30-7. http://dx.doi. org/10.1111/j.1600-051X.2011.01806.x.
27. Choi YH, McKeown RE, Mayer-Davis EJ, Liese AD, Song KB, Merchant AT. Association between periodontitis and impaired fasting glucose and diabetes. Diabetes Care. 2011;34:381-6. http://dx.doi.org/10.2337/dc10-1354.

28. Rivas-Tumanyan S, Spiegelman D, Curhan GC, Forman JP, Joshipura KJ. Periodontal disease and incidence of hypertension in the health professionals follow-up study. Am J Hypertens. 2012;25:770-6. http://dx.doi.org/10.1038/ ajh.2012.32.

29. Hansson GK. Inflammation, atherosclerosis, and coronary artery disease. N Engl J Med. 2005;352:1685-95. http:// dx.doi.org/10.1056/NEJMra043430

30. Iacopino AM. Periodontitis and diabetes interrelationships: Role of inflammation. Ann Periodontol. 2001;6:125-37. http:// dx.doi.org/10.1902/annals.2001.6.1.125.

31. Warnakulasuriya S, Dietrich T, Bornstein MM, Casals Peidro E, Preshaw PM, Walter C, et al. Oral health risks of tobacco use and effects of cessation. Int Dent J. 2010;60:7-30.

32. Bushnell PT, Colombi A, Caruso CC, Tak S. Work schedules and health behavior outcomes at a large manufacturer Ind Health. 2010;48:395-405. http://dx.doi.org/10.2486/ indhealth.MSSW-03.

33. van Amelsvoort LGPM, Schouten EG, Kok FJ. Impact of one year of shift work on cardiovascular disease risk factors. JOEM. 2004;46:699-706. http://dx.doi.org/10.1097/01. jom.0000131794.83723.45.

34. Hiro H, Kawakami N, Tanaka K, Nakamura K. Association between job stressors and heavy drinking: Age differences in male Japanese workers. Ind Health. 2007;45:415-25. http:// dx.doi.org/10.2486/indhealth.45.415.

35. Waterhouse J, Buckley P, Edwards B, Reilly T. Measurement of, and some reasons for, differences in eating habits between night and day workers. Chronobiol Int. 2003;20:1075-92. http://dx.doi.org/10.1081/CBI-120025536.

36. Morikawa Y, Sakurai M, Nakamura K, Nagasawa SY, Ishizaki M, Kido T, et al. Correlation between shift-work-related sleep problems and heavy drinking in japanese male factory workers. Alcohol Alcoholism. 2013 Mar-Apr;48(2):202-6. http://dx.doi.org/10.1093/alcalc/ags128.

37. Antunes L, Levandovski R, Dantas G, Caumo W, Hidalgo M. Obesity and shift work: Chronobiological aspects. Nutr Res Rev. 2010;23:155-68. http://dx.doi.org/10.1017/ S0954422410000016.

38. Costa G, Sartori S. Ageing, working hours and work ability. Ergonomics 2007;50:1914-30. http://dx.doi. org/10.1080/00140130701676054.

Received for publication: 10 January 2013 